\title{
Inverse correlation of Acinetobacter spp. resistance rate and ciprofloxacin utilization
}

\author{
The Journal of Antibiotics (2014) 67, 273-275; doi:10.1038/ja.2013.123; published online 20 November 2013
}

Several studies tried to investigate the relation between utilization rate of antibiotics and resistance, but only some of them found that increased utilization had been associated with increase in resistance to antibiotics. ${ }^{1,2}$ Other studies either did not find a correlation or found a very weak one. ${ }^{3-5}$ In order to clarify the issue further, we have evaluated the impact of antibiotic consumption on resistance rate of multidrugresistant bacteria (Pseudomonas aeruginosa and Acinetobacter spp.) to antibiotics among inpatients from intensive care unit of a tertiary care university hospital 'Clinical Center of Kragujevac', during a 5-year period (2007-2011). Antibiotic utilization rate was expressed in DDDs (defined daily doses) per 100 BDs (bed days), and resistance of isolated bacteria to antibiotics was tested by diskdiffusion method. There was between 600 and 700 isolates per year and 200 of them were randomly selected for each study year; from the 1000 selected isolates, only those of P. aeruginosa (314 isolates) and Acinetobacter spp. (140 isolates) were taken into account (according to the sample size calculation, we needed 141 isolates for establishing resistance rate of $P$. aeruginosa (confidence level 95\%, confidence interval $\pm 7.5 \%$ and resistance rate to ciprofloxacin from previous studies 29\%) and 96 isolates for establishing resistance rate of Acinetobacter spp. (confidence level 95\%, confidence interval $\pm 10 \%$ and resistance rate to ciprofloxacin from previous studies 66\%). ${ }^{6,7}$ Within each of the two bacterial species, all isolates were of the same serological phenotype, implying the same clonal ancestry. The origin of the isolates were as following: $26.5 \%$ were isolated from infected operative wounds, $24.9 \%$ from urine and $19.4 \%$ from tracheal aspirates.

For $P$. aeruginosa, only resistance to piperacillin/tazobactam significantly correlated with utilization rate of this antibiotic $(r=0.952, P=0.013)$ as shown in Table 1 . The resistance rate ranged from $5.1 \%$ to $13.4 \%$, and utilization of piperacillin/tazobactam ranged from 0.12 to 0.34 DDDs per 100 BDs. Resistance rates of Acinetobacter spp. to piperacillin/tazobactam, ciprofloxacin and ceftazidime correlated well with utilization rates of these antibiotics $(r=0.882$, $P=0.048 ; r=-0.913, P=0.030 ; r=-0.991$, $P=0.009$, respectively; Table 1 ), although in opposite directions: for piperacillin/tazobactam the correlation was direct, whereas for the other two antibiotics the correlation was inverse. The resistance rate of Acinetobacter spp. ranged from $8 \%$ to $94 \%$ for piperacillin/tazobactam, from $73 \%$ to $100 \%$ for ciprofloxacin and from $91.3 \%$ to $100 \%$ for ceftazidime, whereas the utilization rate ranged from 4.15 to 5.68 DDDs per 100 BDs for ciprofloxacin and from 0.9 to 2.08 DDDs per 100 BDs for ceftazidime (Table 1).

It was found in some studies that highutilization rate of $\beta$-lactams increased prevalence of $\beta$-lactam-resistant $P$. aeruginosa, and that decreasing $\beta$-lactam utilization was an efficient method to increase susceptibility of $P$. aeruginosa, Acinetobacter spp. and other pathogens to $\beta$-lactam antibiotics. ${ }^{8-10}$ In our study, the same conclusion was confirmed

Table 1 Correlation between antibiotic utilization rates (DDDs per 100 BDs) and resistance rates of $P$. aeruginosa and Acinetobacter spp. (\%); annual antibiotic utilization rates (DDDs per $100 \mathrm{BDs}$ ) and pathogen resistance rates (\%)

\begin{tabular}{|c|c|c|c|c|c|c|c|c|c|}
\hline & & \multicolumn{3}{|c|}{ Piperacillin/tazobactam } & \multicolumn{3}{|c|}{ Ciprofloxacin } & \multicolumn{2}{|c|}{ Ceftazidime } \\
\hline & & \multicolumn{2}{|l|}{$r$} & $\mathrm{P}$-value & $r$ & \multicolumn{2}{|c|}{ P-value } & r & P-value \\
\hline \multirow{2}{*}{\multicolumn{2}{|c|}{$\begin{array}{l}\text { P. aeruginosa } \\
\text { Acinetobacter spp. }\end{array}$}} & 0.952 & \multicolumn{2}{|r|}{0.013} & -0.375 & \multicolumn{2}{|c|}{0.534} & 0.448 & 0.449 \\
\hline & & 0.882 & & 0.048 & -0.913 & \multicolumn{2}{|c|}{0.03} & -0.991 & 0.009 \\
\hline Year & $\begin{array}{l}\text { P. aeruginosa } \\
\text { resistance rates }\end{array}$ & $\begin{array}{c}\text { Acinetobacter spp. } \\
\text { resistance rates }\end{array}$ & $\begin{array}{l}\text { Utilization } \\
\text { rates }\end{array}$ & $\begin{array}{l}\text { P. aeruginosa } \\
\text { resistance rates }\end{array}$ & $\begin{array}{c}\text { Acinetobacter spp. } \\
\text { resistance rates }\end{array}$ & $\begin{array}{l}\text { Utilization } \\
\text { rates }\end{array}$ & $\begin{array}{l}\text { P. aeruginosa } \\
\text { resistance rates }\end{array}$ & $\begin{array}{c}\text { Acinetobacter spp. } \\
\text { resistance rates }\end{array}$ & $\begin{array}{l}\text { Utilization } \\
\text { rates }\end{array}$ \\
\hline 2007 & 6.5 & 8 & 0.12 & 73.6 & 100 & 4.15 & 65.9 & 100 & 0.9 \\
\hline 2008 & 5.1 & 13.6 & 0.16 & 62.2 & 88.1 & 5.14 & 46.9 & 100 & 1.39 \\
\hline 2009 & 10.2 & 49.8 & 0.27 & 56.6 & 80 & 5.68 & 50.9 & 100 & 1.46 \\
\hline 2010 & 12.8 & 53.4 & 0.34 & 77.8 & 73.3 & 5.48 & 76.0 & 91.3 & 2.08 \\
\hline 2011 & 13.4 & 94 & 0.32 & 68.8 & 73 & 5.53 & 67.4 & 92.9 & 1.88 \\
\hline
\end{tabular}

Abbreviations: 100BDs, 100 bed days; DDD, defined daily doses; $r$, Pearson's correlation coefficient. 


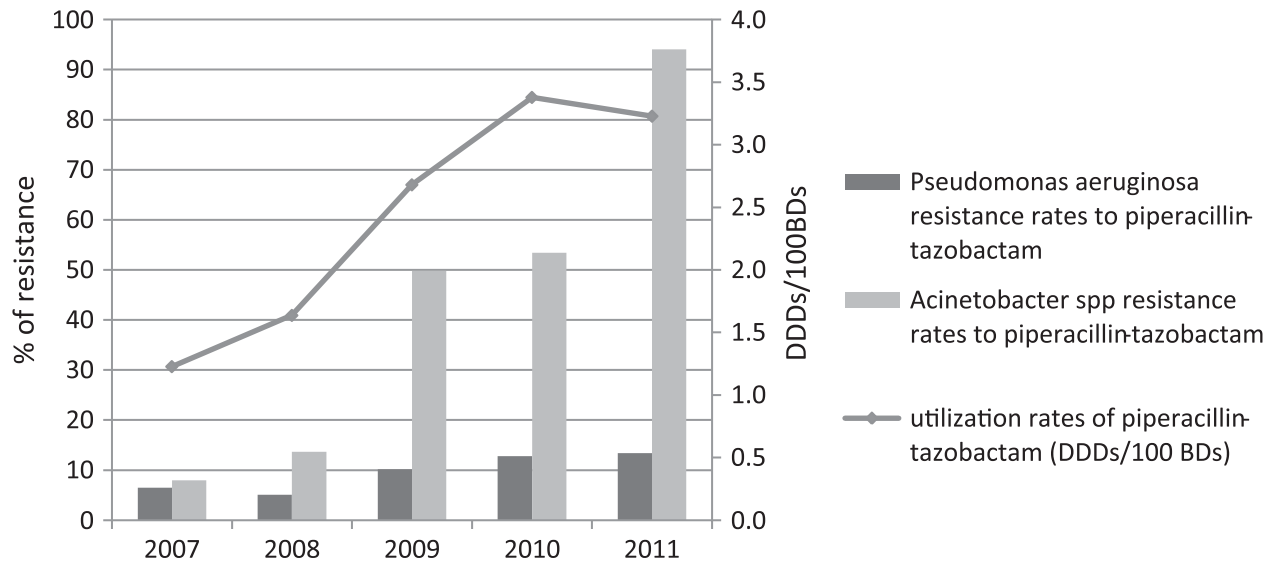

Figure 1 Association between piperacillin/tazobactam utilization rates and resistance rates of $P$. aeruginosa and Acinetobacter spp.

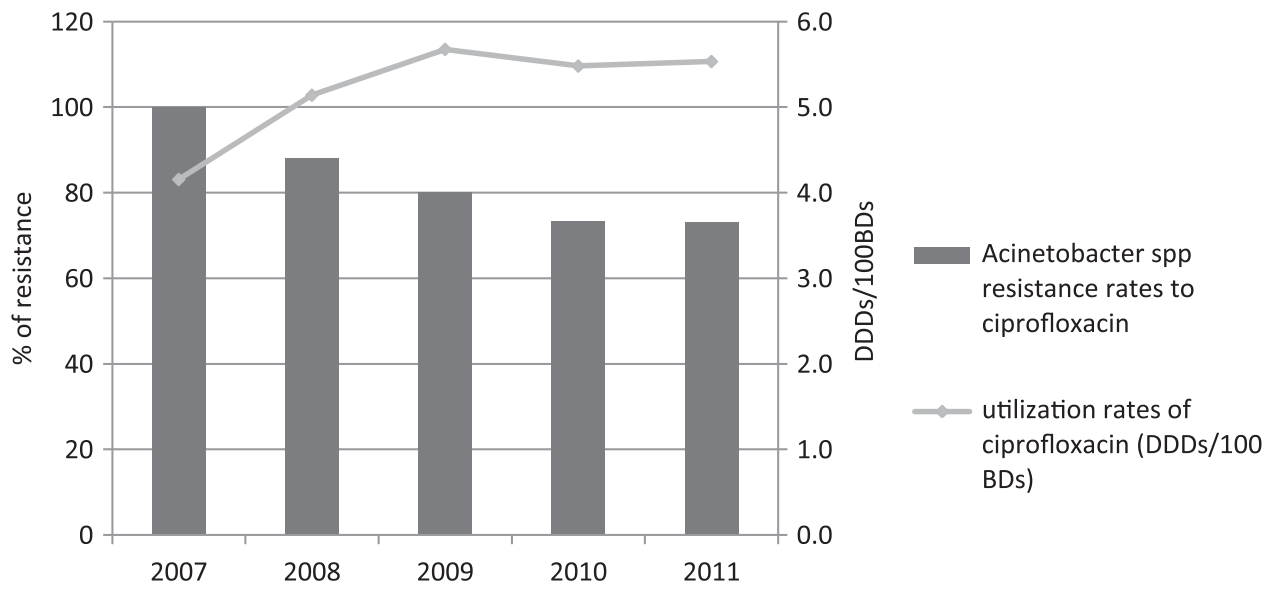

Figure 2 Association between ciprofloxacin utilization rates and resistance rates of Acinetobacter spp.

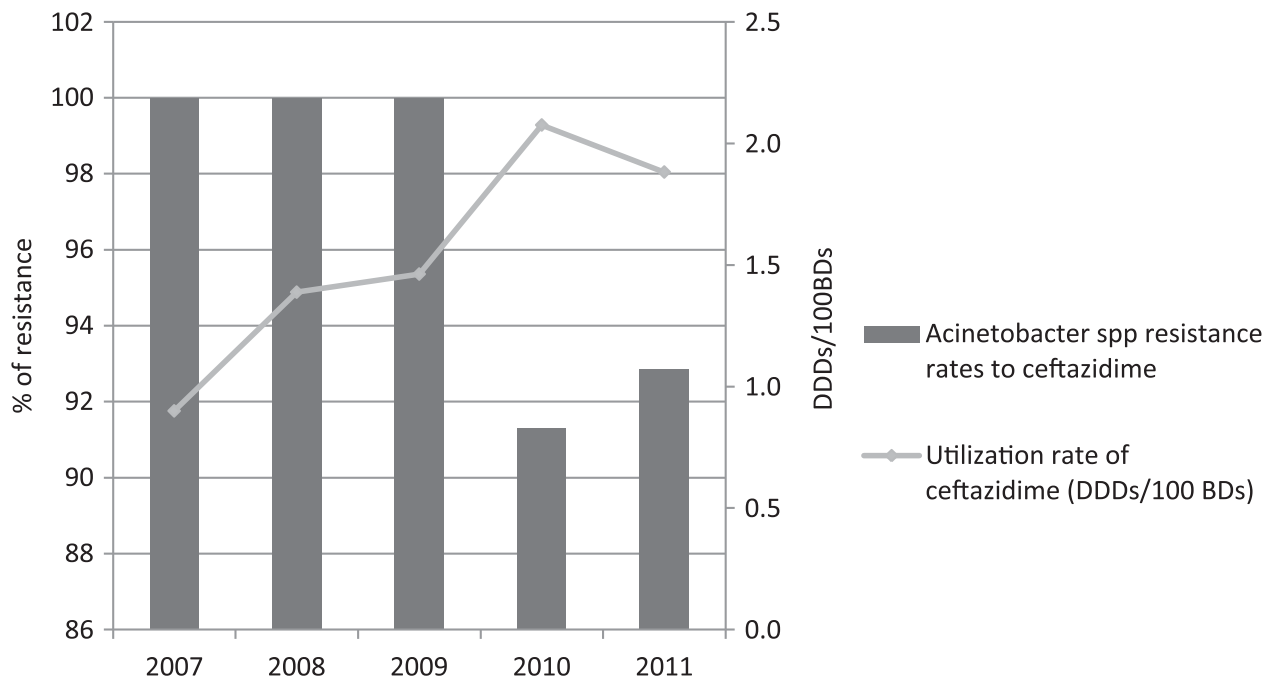

Figure 3 Association between ceftazidime utilization rates and resistance rates of Acinetobacter spp. 
for piperacillin/tazobactam (Figure 1), but the opposite phenomenon was observed with ciprofloxacin, a non- $\beta$-lactam drug (Figure 2), and with ceftazidime (Figure 3), the $\beta$-lactam drug from cephalosporin subgroup. Although direct correlation between $P$. aeruginosa resistance rate and utilization of fluoroquinolones was found previously, ${ }^{2}$ the same was never shown for Acinetobacter spp. and this group of antibiotics. ${ }^{4}$

One of the most important adaptive mechanisms of bacterial resistance to antibiotics is efflux pumps, which could be either overexpressed or inhibited by specific compounds with similar chemical structure as antibiotics. ${ }^{11,12}$ Whereas gradually increasing utilization of antibiotics, which are administered as monotherapy (like piperacillin/tazobactam in our study), progressively selects resistant strains with overexpressed efflux pumps and increases overall rate of resistance to antibiotics, constant high-utilization rate of antibiotics, which are part of combination regimens (like ciprofloxacin and ceftazidime), could saturate the efflux pumps and make the bacteria more susceptible for the antibiotics concomitantly given to the patient. Ciprofloxacin and ceftazidime were never given as monotherapy in our intensive care unit: ceftazidime was usually combined with aminoglycosides, whereas ciprofloxacin frequently accompanied third-generation cephalosporins.
Although our data are limited, and are not fully supportive of our hypothesis, it is clear that both extent and pattern of antibiotic utilization are important determinants of antibiotic resistance. Many circumstances, including number, type, dosage and duration of antibiotic therapy could influence development of bacterial resistance, which could even decrease in spite of high overall antibiotics utilization figures.

\section{ACKNOWLEDGEMENTS}

This work was partially supported by grant no 175007 from the Serbian Ministry of Education, and by a grant given by the Ministry of Science, Montenegro. The authors are also grateful to Dragana Nedovic, Sladjana Petrovic and Nebojsa Milivojevic for collecting the data.

Radisa R Pavlovic and Slobodan M Jankovic

Departament of Clinical Pharmacology, Clinical Center and Faculty of Medical Science, Kragujevac, Serbia E-mail: rpavlovic@medf.kg.ac.rsor slobodan.jankovic@medf.kg.ac.rs

1 Goossens, H. et al. Outpatient antibiotic use in Europe and association with resistance: a cross-national database study. Lancet 365, 579-587 (2005).

$2 \mathrm{Wu}, \mathrm{H} . \mathrm{-H}$. et al. Correlation between levofloxacin consumption and the incidence of nosocomial infections due to fluoroquinolone-resistant Escherichia coli. J. Microbiol. Immunol. Infect. (e-pub ahead of print 4 May 2012; doi:10.1016/j.jmii.2011. 12.019).

3 Bergman, M. et al. Association between antimicrobial consumption and resistance in Escherichia coli. Antimicrob. Agents Chemother. 53, 912-917 (2009).

4 Bartoloni, A. et al. High prevalence of acquired antimicrobial resistance unrelated to heavy antimicrobial consumption. J. Infect. Dis. 189, 1291-1294 (2004).

5 Ho, C.-M. et al. Correlation between carbapenem consumption and resistance to carbapenems among Enterobacteriaceae isolates collected from patients with intra-abdominal infections at five medical centers in Taiwan, 2006-2010. Int. J. Antimicrob. Agents 40, 24-28 (2012).

6 Dogru, A. et al. The rate of device-associated nosocomial infections in a medical surgical intensive care unit of a training and research hospital in Turkey: one-year outcomes. Jpn J. Infect. Dis. 63, 95-98 (2010).

7 Shakibaie, M. R., Adeli, S. \& Salehi, M. H. Antibiotic resistance patterns and extended-spectrum $\beta$-lactamase production among Acinetobacter spp. isolated from an intensive care unit of a hospital in Kerman, Iran. Antimicrob. Resist. Infect. Control 1, 1 (2012).

8 Miyawaki, K. et al. Correlation between the consumption of meropenem or doripenem and meropenem susceptibility of Pseudomonas aeruginosa in a university hospital in Japan. Biol. Pharm. Bull. 35, 946-949 (2012).

9 Nakamura, A. et al. Association between antimicrobial consumption and clinical isolates of methicillin-resistant Staphylococcus aureus: a 14-year study. J. Infect. Chemother. 18, 90-95 (2012).

$10 \mathrm{Goel}, \mathrm{N}$. et al. Trend analysis of antimicrobial consumption and development of resistance in non-fermenters in a tertiary care hospital in Delhi, India. J. Antimicrob. Chemother. 66, 1625-1630 (2011).

11 Lomovskaya, O. \& Watkins, W. Inhibition of efflux pumps as a novel approach to combat drug resistance in bacteria. J. Mol. Microbiol. Biotechnol. 3, 225-236 (2001).

12 Rodríguez-Martínez, J. M., Poirel, L. \& Nordmann, P. Molecular epidemiology and mechanisms of carbapenem resistance in Pseudomonas aeruginosa. Antimicrob. Agents Chemother. 53, 4783-4788 (2009). 